CERN LIBRARIES, GENEVA

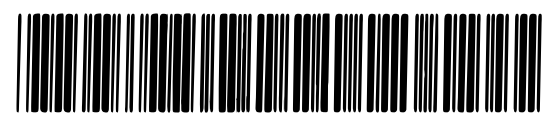

CM-P00064341

EUROPEAN ORGANIZATION FOR NUCLEAR RESEARCH

CERN-ISR-GE/ 77-63

M. G. DOMIE

III

Closed distribution

\title{
A TITANIUM HIGH PRESSURE GAS TARGET
}

by

$\mathrm{J}-\mathrm{C}$. Brunet and C. Hauviller

\section{Abstract}

Design, manufacturing and testing of a titanium high pressure (150 bars) gas target developped at CERN is presented.

(submitted to "Nuclear Instruments and Methods")

Geneva, October 1977 
Gas targets are used in a fairly large number of high energy physics experiments, especially when the target recoil particles are to be detected at low energies.

In order to reduce energy loss, multiple Coulomb scattering, and the number of secondary events, one must design a cylindrical container for the target gas which has a very transparent wall.

The transparency of a wall is related to $L_{c} t$ and $L_{R} t, t$ being the thickness of the wall and $L_{c}$ and $L_{R}$ the collision length and radiation length of the wall material. But, on the other hand, the pressure inside the target must be maximized; for a cylinder, the elasticity condition imposes that (for $t \ll R$ )

$$
p=\frac{\sigma_{0,2}}{S} \frac{t}{R}
$$

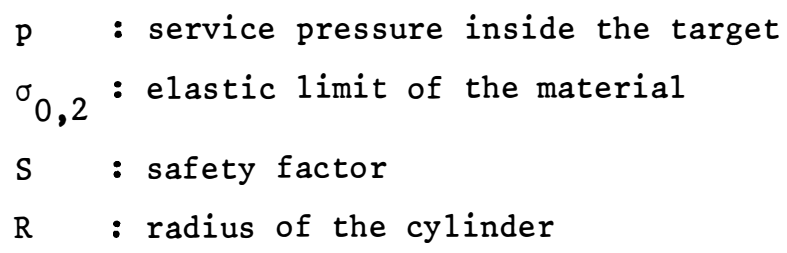

Then, using this last relation for a given pressure and geometry, one finds that the transparency is related to $\mathrm{L}_{c} \sigma_{0,2}$ and $\mathrm{L}_{\mathrm{R}} \sigma_{0,2}$ and that its maximum value is obtained when these products are maximum.

Among the different types of technically reliable materials, titanium alloys have the best performances for high pressures $\left(\sigma_{0,2}>80\right.$ hbar at $20^{\circ} \mathrm{C}, \mathrm{L}_{\mathrm{C}} \curvearrowright 21 \mathrm{~cm}$ and $\left.L_{R} \sim 3,5 \mathrm{~cm}\right)$.

The target is a cylindrical tube of $2 \mathrm{~cm}$ diameter and length $58 \mathrm{~cm}$. The $250 \mu \mathrm{m}$ thick cylindrical wall is manufactured in titanium alloy (13\% vanadium, $11 \%$ chromium, $3 \%$ aluminium) with a minimum elastic limit $\sigma_{0,2}=84$ hbar at $20^{\circ} \mathrm{C}$. The ends of the cylinder are closed by two hemispherical caps machined in commercially pure titanium $\left(\sigma_{0,2}=37\right.$ hbar $)$; the thickness varies from $400 \mu \mathrm{m}$ on the axis to $600 \mu \mathrm{m}$ at the connection to the cylinder. 
The structure is self-supporting, stable under vacuum and homogeneous from a stress point of view. One branch connection is used to put the target under vacuum or pressure.

The forming and welding processes were of a delicate nature. A good reliable result was, however, obtained owing to precise tooling and systematic $\mathrm{X}$-ray tests along with micro-hardness, elongation and elastic limit measurements.

Five identical targets were manufactured; two of them, equipped with strain gauges were tested up to bursting pressure in order to verify stress computations and manufacturing quality. In the titanium alloy parts, the elastic limit at 0,2\% was reached at a pressure of 192 bars, compared with a computed value of 210 bars. This means that the elastic limit in the welds was only $10 \%$ below that of the parent metal. In the pure titanium parts, the elastic limit was reached at a pressure greater than 250 bars. These two targets exploded at 280 and 290 bars respectively. The safety factor $S$ was taken equal to 1.25. So the maximum service pressure was fixed at 150 bars, which is ten times larger than the usual pressure for this type of target.

One of these targets (see picture) was used (1) with success under a pressure of 150 bars, full of hydrogen during five days, then full of helium during a fortnight. About ten pressurizations were carried out.

Such a target has many interesting features. In particular, filled with hydrogen, it is equivalent, from the point of view of material, to a liquid hydrogen target of $11 \mathrm{~cm}$ thickness. However, the quantity of material used for the containment is very small and the signal to noise ratio is excellent. Finally, one must note that the physicists can easily measure the background noise associated with the experiment by putting the target under vacuum.

The authors are indebted to the ISR workshop at CERN, especially A. Ninet, for the high quality of the work. 


\section{REFERENCE}

(1) CERN Experiment S 143

Clermont-Ferrand - Lyon - Lund - Uppsala - Warsaw Collaboration. 
General view of high-pressure gas target.

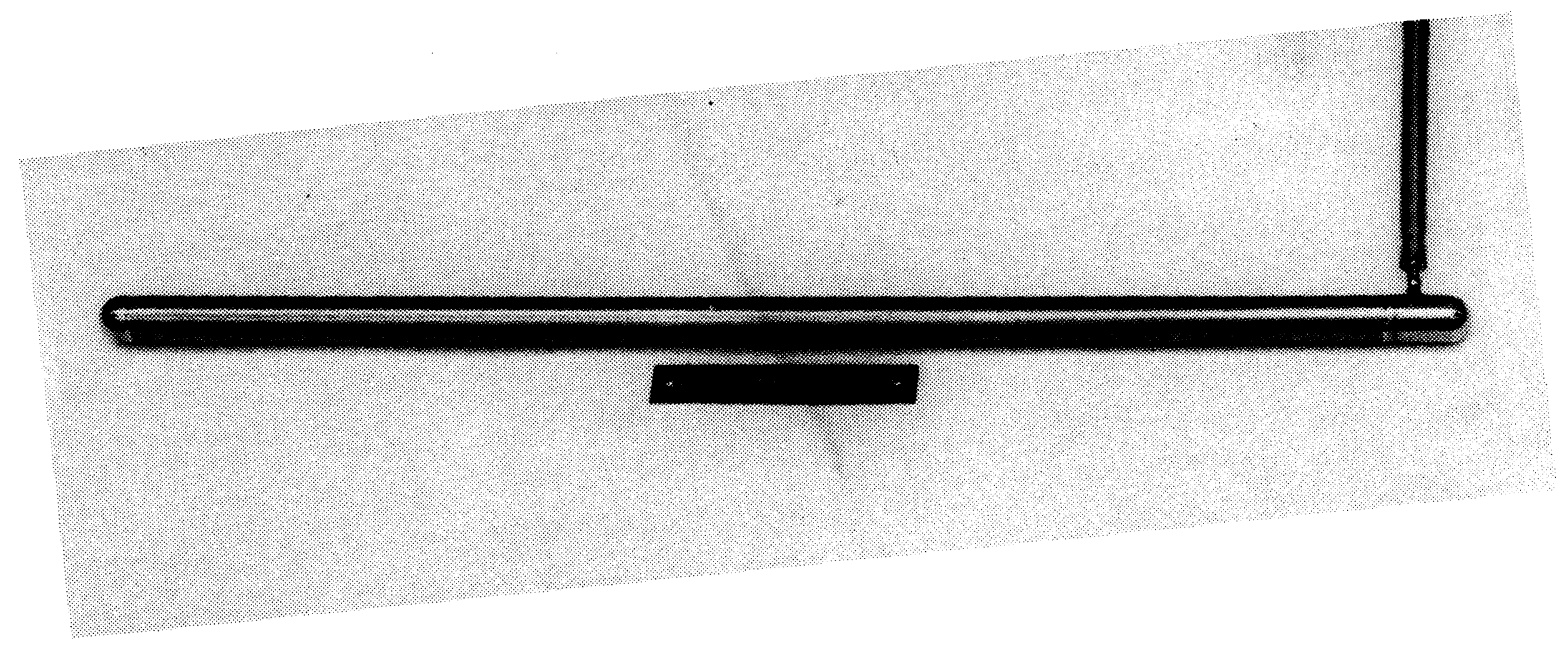

